\title{
Non-specific fluorescent whitener stains in the rapid recognition of pulmonary dirofilariasis: a report of 20 cases
}

\author{
Linda K Green, M Qasim Ansari, Mary R Schwartz, Jae Y Ro, Lesley C Alpert
}

\begin{abstract}
Background - Solitary lung nodules in humans caused by the dog parasite Diroflaria immitis are steadily increasing in number. The organisms can be easily missed in haematoxylin and eosin stained tissue when they are degenerated and pale staining.

Methods - The value of Tinopal CBS-X (TCBS-X) and Calcofluor white (CFW), two rapid, inexpensive, simple nonspecific fluorescent whitening stains, were assessed in the identification of these worms. Deparaffinised rehydrated tissue slides prepared from the pulmonary nodules were stained for one minute in $1 \% \mathrm{w} / \mathrm{v}$ aqueous solutions of TCBS-X or CFW, counterstained, coverslipped, and viewed with a fluorescent microscope.

Results - The stains demonstrated the intact worm and worm fragments in $\mathbf{2 0}$ cases of pulmonary dirofilariasis collected from hospitals in Houston. The filariae and fragments of filariae stained bright green while the background tissue stained red, delineating the internal structures of the worm.

Conclusions - Dirofilariasis should be included in the differential diagnosis of subpleural masses, and non-specific fluorescent whitening stains can help in the rapid recognition of the fragmented organism in cytological or surgical material.
\end{abstract}

(Thorax 1994;49:590-593)

The incidence of human pulmonary dirofilariasis has been increasing over the last three decades. ${ }^{1-3}$ This zoonosis caused by the dog heartworm, Dirofilaria immitis, was confined for many years to a coastal belt from New York extending south to the Gulf coast. In recent years it has extended to the Mississippi River Valley, southern Canada, and sporadically throughout the rest of the United States. ${ }^{45}$

Dirofilaria immitis, a filarial nematode, is transmitted from dogs to humans by mosquitoes who serve as an intermediate host and vector. Because man is an accidental and imperfect host, the parasite cannot mature into an adult worm and presents in a filarial form as a subcutaneous or pulmonary nodule. ${ }^{6}$ The pulmonary lesion may mimic a neoplasm both clinically and radiologically, and a fine needle aspiration or thoracotomy may be required for diagnosis. ${ }^{7}$ In many of these lesions the parasite may be difficult to recognise in histological or cytological material because of degeneration or fragmentation of the organism.

There is a need for a rapid and reliable method of identifying this parasite on frozen section, fine needle aspiration, and permanent formalin fixed, paraffin embedded tissue. Nonspecific fluorescent whitener stains such as Tinopal CBS-X (TCBS-X) and Calcofluor white (CFW) have been shown to stain nematodes and other parasites by binding to their external chitin walls. ${ }^{8}$ We found that TCBS-X and CFW stained the intact organism and parasitic fragments intensely, allowing rapid identification. The staining was also useful in accentuating the internal structure of the filaria, thus aiding classification. This study presents the clinical, radiological, and pathological findings of 20 patients with pulmonary dirofilariasis and illustrates the usefulness of non-specific whitener stains (TCBS-X and CFW) for identifying the organism.

\section{Methods}

Twenty cases of pulmonary dirofilariasis were identified from a retrospective and prospective review of the surgical files at the Baylor College of Medicine Affiliated Hospitals (Veterans Affairs Medical Center, Methodist Hospital, and Ben Taub Hospital) (16 cases) and the UTMD Anderson Cancer Center (four cases) in Houston, Texas from 1965 to 1993. Three of the four cases from M D Anderson were previously reported in 1989. All cases were pulmonary wedge resections of lung nodules. In each case 1-10 tissue sections (average three) with 1-3 step sections from each block were stained with haematoxylin and eosin. Selected tissue sections were stained with the Gomori methenamine silver (GMS) method, the Periodic acid-Schiff (PAS) reaction with or without previous diatase digestion, and the Ziehl-Neelson method. The clinical records, radiological, and histological findings of the patients were reviewed.

Staining with non-specific fluorescent whiteners was performed on formalin fixed, paraffin embedded tissue in all cases. Fresh tissue from six cases was frozen in optimal cutting temperature embedding medium, sectioned, and fixed in ethanol. One step section was cut from each block containing the lesion. Deparaffinised unstained tissue slide sections were rehydrated and stained with stock solutions $(0.1 \% \mathrm{w} / \mathrm{v})$ of TCBS-X (Ciba-Geigy, Greensboro, North Carolina, USA) or CFW 
(Fungi-Fluor, Polyscience, Warrington, Pennsylvania, USA), both derivatives of diaminostilbene disulphonic acid. ${ }^{8}$ The rehydrated slides were stained for two minutes in the TCBS-X or CFW solutions, rinsed with tap water, counterstained with Evan's blue, and examined with a fluorescent microscope using a Nikon B-1E exciter filter.

\section{Results}

CLINICAL FINDINGS

There were 20 patients (six women) of mean age 58 (range 35-77) years. Eleven cases presented with subpleural nodules and nine had round lesions or nodules within the parenchyma. All lived in the south-western United States, mostly in Texas. The pulmonary nodules were asymptomatic in 17 patients, one had localised retrosternal chest pain, and none had peripheral eosinophilia. In the asymptomatic patients the lesions were found on routine chest radiographs. Eleven patients were smokers and one had an asbestos exposure.

Chest radiography or computed tomographic scans revealed the nodule to be single in 18 cases, ranging in size from 0.8 to $4.5 \mathrm{~cm}$ in diameter. In one case there were two nodules, each measuring $0.5 \mathrm{~cm}$ in diameter. Radiographically none of the nodules were calcified and all were peripheral in location; 10 were subpleural. The lesions occurred in all lobes except for the right middle lobe. In all of the patients the radiological and clinical findings were indistinguishable from a malignant lesion.

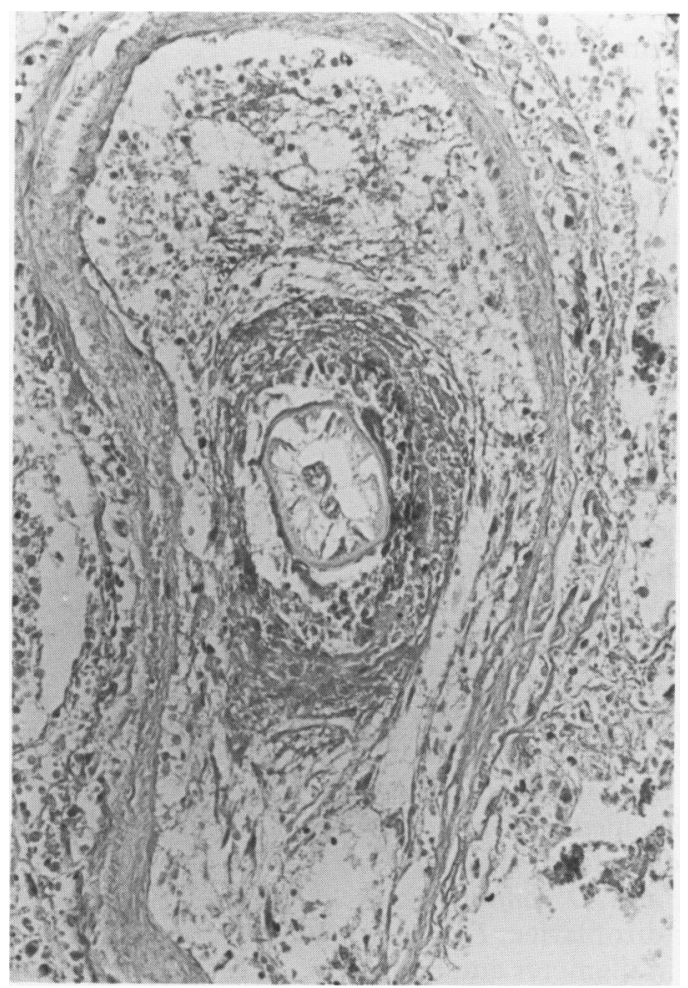

Figure $1 A$ small arteriole within the centre of a round pulmonary lesion showing intimal proliferation and ischaemic necrosis. A cross section of a degenerating worm is present within the lumen. Stain: haematoxylin and eosin; original magnificatioin $\times 300$, reduced to $78 \%$ during origination.
Bronchoscopic examination was normal in all patients, but fine needle aspiration cytology in one patient was initially interpreted as being suspicious for malignancy. All of the patients had uneventful postoperative courses following thoracotomy.

\section{PATHOLOGICAL FINDINGS}

On gross examination the lesions were grey to yellow and relatively well circumscribed. On cut section they were firm, granular nodules with central softening and variegation. In a few cases the adjacent lung was firm to rubbery. Histological examination with haematoxylin and eosin showed features of a circumscribed area of pulmonary infarction or necrosis with focally preserved alveolar wall silhouettes and scattered inflammatory cells. The sharply defined periphery comprised palisading epithelioid histiocytes, lymphocytes, and occasional multinucleated giant cells. Adjacent lung parenchyma contained mixed cellular infiltrates including plasma cells and rare eosinophils. Preserved vessels showed prominent intimal proliferation. In each of the lesions haematoxylin and eosin stained section contained small arterioles with cross sections or often unrecognised fragments of coiled parasites within their lumens (fig 1). In many cases the parasites stained pale pink and blended into the pink, necrotic background. The parasites measured $120-260 \mu \mathrm{m}$ in diameter on cross section with a thick cuticle and lateral cords compressed between heavy muscle bands and central tubular structures. The PAS stain accentuated the somatic muscle while the GMS stain outlined the cuticle in most of the cases.

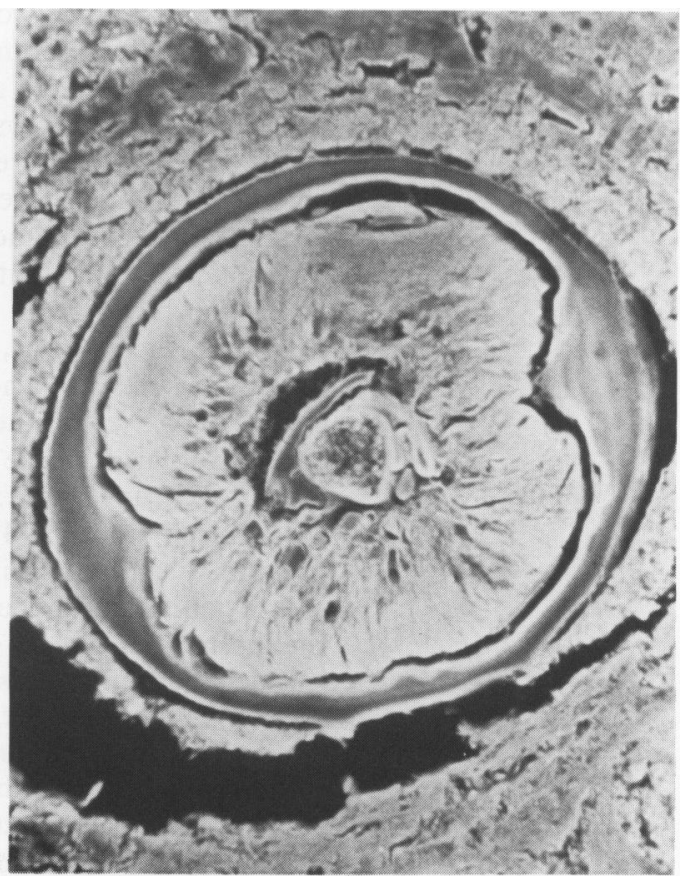

Figure 2 Cross section of an intact worm of D immitis within a small arteriole in the centre of a pulmonary lesion when viewed with a fluorescent microscope. Stain: Tinopal CBS-X; original magnification $\times 1000$, reduced to $59 \%$ during origination. 
FINDINGS AT FLUORESCENT MICROSCOPY

Several sections from each of the 20 cases stained with TCBS-X and CFW contained bright "apple green" staining structures when viewed on low power $(40 \times)$. The structures were seen shining brightly amid a background of red staining necrotic and viable tissue when viewed by fluorescent microscopy. On higher power these were identifiable as portions of either well preserved (fig 2) or necrotic or calcified worms. The cuticle had three distinct layers with crested thickenings at the lateral cord positions, and the somatic muscle bands stained brightly. Centrally, the general cavity or pseudocele contained well delineated tubular structures identifiable as the intestine and sex organs. Cross sections of worms were clearly evident and the stains highlighted fragments of worms not identifiable on routine stains.

\section{Discussion}

Pulmonary dirofilariasis has been sporadically reported in over 100 patients in the United States and it is increasing in incidence. ${ }^{12-59-30} \mathrm{It}$ is clinically important because of its radiological resemblance to a primary bronchogenic carcinoma. ${ }^{7}$ Diagnosis is usually made at excision, though it has been diagnosed by fine needle aspiration cytology. ${ }^{10161931}$

In dogs $D$ immitis causes morbidity and mortality and is an increasing problem. ${ }^{411}$ The adult heartworms reside in the right ventricle and pulmonary artery of dogs which act as a reservoir and is the principal host of the parasite. It is transmitted by the Aedes genus of mosquito which acts as the intermediate host and vector. ${ }^{6}$

A lesion in the lung is formed when the dead worm triggers an Arthus phenomenon. The lesion(s) appears as a round, subpleural dense nodule, and $40-50 \%$ of patients may present with clinical symptoms including chest discomfort, malaise, low grade fever, cough, and occasionally haemoptysis. ${ }^{2}$

Non-specific fluorescent whiteners are chemicals which have been used for many years in the textile and detergent industry as "whiteners" or "brighteners." Both CFW and TCBS-X have been used clinically in the detection of bacteria, fungi, parasites, and pneumocystis. ${ }^{832-34}$ The stains bind tightly to carbohydrate constituents of the organisms. The dirofilaria larvae have a cuticle which contains chitin, a polymer of $N$-acetyl-D-glucosamine in beta (1-4) linkage, which is the presumed site of binding of the stains. Fluorescent whiteners are useful as they delineate the internal structures of the worm even when it is degenerating. This aids identification of $D$ immitis from the other dirofilaria worms ( $D$ repens, $D$ ursi, $D$ tenuis, and $D$ conjunctiva). Compared with the other dirofilariae, $D$ immitis measures $100-300 \mu \mathrm{m}$ in diameter and has a smooth non-ridged multilayered cuticle, 10 $25 \mu \mathrm{m}$ thick, with diametrically opposed internal longitudinal ridges formed by lateral cords, thick bands of somatic muscle, and a central digestive tube. In contrast, the other dirofilar- iae differ in size, have ridged cuticles, and differing amounts of musculature. ${ }^{6}$

Non-specific fluorescent whitening stains aid in distinguishing the organism or its fragments from granulomas, especially in older lesions when organisation of the lesion occurs. The diagnosis relies entirely on identifying the parasite within vascular lumens. In six of our cases the worm would have been disregarded as only debris if the non-specific fluorescent whitening stains had not been used. TCBS-X and CFW make the identification of the worm simple. Non-specific fluorescent whitening stains are rapid, inexpensive and easy to use on granuloma-like pulmonary nodules. They can be easier to obtain than conventional stains (PAS, Trichrome), especially at the time of frozen section, and are easier to interpret. They may also demonstrate bacteria or fungi and are thus multipurpose stains. They do not produce non-specific staining of normal structures when the correct counterstain (Evan's blue) is used. This decreases the problem often encountered in the GMS and PAS stained sections in which collagen and elastin must be distinguished from fragments of the parasite.

1 Adkins RB, Dao AH. Pulmonary dirofilariasis: a diagnostic challenge. South Med $\mathcal{f}$ 1984;77:372-4.

2 Ciferri F. Human pulmonary dirofilariasis in the United States: a critical review. $\mathrm{Am} \quad \mathrm{f}$ Trop Med Hyg 1982;31:302-8.

3 Ciferri F. Human pulmonary dirofilariasis in the West. West f Med 1981;134:158-62.

4 Merrill JR, Otis J, Logan WD, Davis B. The dog heartworm (Dirofilaria immitis) in man: an epidemic pending or in progress? fAMA 1980;243:1066-8.

5 Navarrette AR. Pulmonary dirofilariasis. Chest 1972;61: 51-5.

6 Gutierrez Y. Diagnostic features of zoonotic filariae in tissue sections. Hum Pathol 1984;15:514-25.

7 Toomes H, Delphendahl A, Manke H-G, Vogt-Moykoph I The coin lesion of the lung: a review of 955 resected coin lesions. Cancer 1983;51:534-7.

8 Green LK, Cowan DF, Moore DG. Examination of tissue for parasites by fluorescent microscopy. Histopathology 1988;12:553-5.

9 Caravelli JF, Zaman MB, Bains MS. Human pulmonary dirofilariasis mimicking metastatic disease. Clin Bull 1981;2:88-90.

10 Chesney TM, Martinez LC, Painter MW. Human pulmonary dirofilarial granuloma. Ann Thorac Surg 1983;36: 214-7.

11 Cook GC. Canine-associated zoonoses: an unacceptable hazard to human health. $Q \mathcal{F}$ Med 1989;70:5-26.

12 Dayal Y, Neafie RC. Human pulmonary dirofilariasis: a case report and review of the literature. Am Rev Respir Dis 1975;112:437-43.

13 Feld H. Dirofilaria immitis (dog heartworm) as a cause of a pulmonary lesion in man. Diagn Radiol 1973;108:311-2.

14 Gershwin LJ, Gershwin ME, Kritzman J. Human pulmonary dirofilariasis. Chest 1974;66:92-6.

15 Harrison EG, Thompson JH. Dirofilariasis of human lung. Am 7 Clin Pathol 1965;43:224-34.

16 Hawkins AG, Hsui JG, Smith RM, Stitik FP, Siddiky MA Edwards OE. Pulmonary dirofilariasis diagnosed by fin needle aspiration biopsy. Acta Cytol 1985;29:19-22.

17 Hoch WS, Wershba ME, Patchefsky AS. Pulmonary dirofilariasis. $\mathcal{F}$ Thorac Cardiovasc Surg 1974;67:159-63.

18 Jagusch MF, Roberts RM, Rea HH, Priestley DRA. Human pulmonary dirofilariasis. $N Z$ Med $\mathcal{f} 1984 ; 22: 556-8$.

19 Kelley WT, Firouz-Abadi AA, Roszkowski A, Zimmerman PV. Pulmonary dirofilariasis diagnosed by computerized tomography scan controlled percutaneous needle aspiratomography scan controlled percutan
tion. Aust NZ $¥$ Med 1985;15:656-7.

20 Kochar AS. Human pulmonary dirofilariasis: report of three cases and brief review of the literature. $\mathrm{Am} \mathcal{F} \mathrm{Clin}$ Pathol 1985;84:19-23.

21 Levine SE, Mossler JA, Woodard BH. Dirofilaria immitis: zoonosis of clinical concern. South Med $\mathcal{F}$ 1980;73:749-50.

22 Levinson ED, Ziter FMH, Westcott JL. Pulmonary lesion due to Dirofilaria immitis (dog heartworm): report of four cases with radiologic findings. Radiology 1979;131:305-7.

23 Lewis JF, Williams RW, Tinsley EA. Pulmonary "coin" lesion due to Dirofilaria immitis: report of two cases. Arch Surg 1969;98:388-90.

24 Neafie RC, Piggott J. Human pulmonary dirofilariasis. Arch Pathol 1971;92:342-9. 
25 Prioleau WH, Parker EF, Bradham RR, Gregorie HB. Dirofilaria immitis (dog heartworm) as a pulmonary lesion in humans. Ann Thorac Surg 1976;21:382-5.

26 Risher WH, Crocker EF, Beckman EN, Blalock JB, Ochsner JL. Pulmonary dirofilariasis: the largest single institution experience. $f$ Thorac Cardiovasc Surg 1989;97:303-8

27 Ro JY, Panagiotis JT, White VA, Luna MA, Chang-Tung EG, Green L, et al. Pulmonary dirofilariasis: the great imitator of primary or metastatic lung tumors. A clinicopathologic analysis of seven cases and a review of the literature. Hum Pathol 1989;20:69-76.

28 Robinson NB, Chavez CM, Conn JH. Pulmonary dirofilariasis in man: a case report and review of the literature. $\mathcal{f}$ Thorac Cardiovasc Surg 1977;74:403-8.

29 Schlotthauer JC, Harrison EG, Thompson JH. Dirofilaria- sis: an emerging zoonosis? Arch Environ Health 1969;19:887-90.

30 Tannehill AW, Hatch HB. Coin lesions of the lung due to Dirofilaria immitis. Dis Chest 1968;53:369-71.

31 Roussel F, Delacille A, Campos H, Benozio M, Brasseur P. Fine needle aspiration of retroperitoneal human dirofilariasis with a pseudotumoral presentation. Acta Cytol 1990;34:533-5

32 Green LK, Moore DG. Fluorescent compounds that nonspecifically stain fungi. Lab Med 1987;18:456-9.

33 Monheit JG, Brown G, Kott MM, Schmidt WA, Moore M fungi in cytopathology. Am f Clin Pathol 1986;85:222-5.

34 Monheit JG, Cowan DF, Moore DG. Rapid detection of fungi in tissues using calcofluor white and fluorescent fungi in tissues using calcofluor white and fluorescent microscopy. Arch Pathol 1984;108:616-8. 